\title{
ARTICLE \\ Baicalin prevents LPS-induced activation of TLR4/NF-kB p65 pathway and inflammation in mice via inhibiting the expression of CD14
}

\author{
Ya-jun Fu' ${ }^{1}$, Bo Xu ${ }^{1}$, Shao-wei Huang ${ }^{1}$, Xia Luo ${ }^{1}$, Xiang-liang Deng ${ }^{1,2,3}$, Shuang Luo ${ }^{1}$, Chang Liu ${ }^{1}$, Qing Wang ${ }^{1}$, Jin-yan Chen ${ }^{4}$ and \\ Lian Zhou $^{1}$
}

Previous studies have shown that baicalin, an active ingredient of the Chinese traditional medicine Huangqin, attenuates LPSinduced inflammation by inhibiting the activation of TLR4/NF-KBp65 pathway, but how it affects this pathway is unknown. It has been shown that CD14 binds directly to LPS and plays an important role in sensitizing the cells to minute quantities of LPS via chaperoning LPS molecules to the TLR4/MD-2 signaling complex. In the present study we investigated the role of CD14 in the antiinflammatory effects of baicalin in vitro and in vivo. Exposure to LPS $(1 \mu \mathrm{g} / \mathrm{mL})$ induced inflammatory responses in RAW264.7 cells, evidenced by marked increases in the expression of MHC II molecules and the secretion of NO and IL-6, and by activation of MyD88/NF-KB p65 signaling pathway, as well as the expression of CD14 and TLR4. These changes were dose-dependently attenuated by pretreatment baicalin $(12.5-50 \mu \mathrm{M})$, but not by baicalin post-treatment. In RAW264.7 cells without LPS stimulation, baicalin dose-dependently inhibit the protein and mRNA expression of CD14, but not TLR4. In RAW264.7 cells with CD14 knockdown, baicalin pretreatment did not prevent inflammatory responses and activation of MyD88/NF-KB p65 pathway induced by high concentrations $(1000 \mu \mathrm{g} / \mathrm{mL})$ of LPS. Furthermore, baicalin pretreatment also inhibited the expression of CD14 and activation of MyD88/NF-KB p65 pathway in LPS-induced hepatocyte-derived HepG2 cells and intestinal epithelial-derived HT-29 cells. In mice with intraperitoneal injection of LPS and in DSS-induced UC mice, oral administration of baicalin exerted protective effects by inhibition of CD14 expression and inflammation. Taken together, we demonstrate that baicalin pretreatment prevents LPS-induced inflammation in RAW264.7 cells in CD14-dependent manner. This study supports the therapeutic use of baicalin in preventing the progression of LPS-induced inflammatory diseases.

Keywords: inflammatory diseases; baicalin; CD14; TLR4; NF-kB p65; RAW264.7 cells

Acta Pharmacologica Sinica (2021) 42:88-96; https://doi.org/10.1038/s41401-020-0411-9

\section{INTRODUCTION}

Lipopolysaccharide (LPS) from gram-negative bacteria is a pathogen-associated molecular pattern (PAMP) that causes serious inflammatory injury and even death. LPS induces inflammatory injury involving the TLR4/NF-KB p65 inflammatory signaling pathway. It has been demonstrated that TLR4 is the receptor for LPS. After TLR4 encounters LPS, it activates the MyD88-dependent signaling pathway, triggers the NF-kB p65 transcription response, and induces proinflammatory cytokine secretion in macrophages, dendritic cells and some epithelial cells [1]. Thus, many anti-inflammatory drugs, including ingredients from traditional Chinese medicine, target the activation of the TLR4/NF-KB p65 inflammatory signaling pathway.

Huangqin (Scutellaria radix) is widely used in traditional Chinese medicines for the clinical treatment of inflammatory diseases. Baicalin is one of the main active ingredients in Huangqin and has remarkable anti-inflammatory activities in treating inflammatory bowel diseases, rheumatoid arthritis, autoimmune hepatitis and chronic liver diseases [2]. One of the mechanisms by which baicalin exerts its anti-inflammatory effects is by attenuating the activity of NF-kB p65 and suppressing the expression of inflammatory cytokines and other inflammatory mediators. For example, in vitro and in vivo studies have demonstrated that baicalin attenuates LPS-induced inflammation by inhibiting activation of the MyD88/NF-KB p65 pathway and the subsequent secretion of IL-1 $\beta, I L-6, N O$, and TNF- $\alpha$ [3-5]. However, how baicalin inhibits activation of the TLR4/NF-KB p65 pathway is largely unknown.

The TLR4/NF-KB pathway plays an important role in not only inflammation but also the survival, proliferation and immune regulation of cells. It is very interesting that baicalin alleviates LPSinduced inflammation by inhibiting the TLR4/NF-KB pathway but has little effect on survival or proliferation in macrophages. This indicates that the anti-inflammatory activity of baicalin may not directly target the TLR4/NF-KB pathway. The cell surface protein

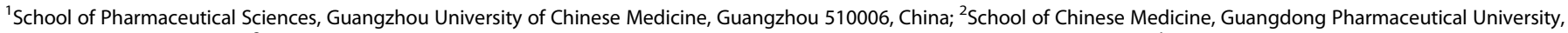

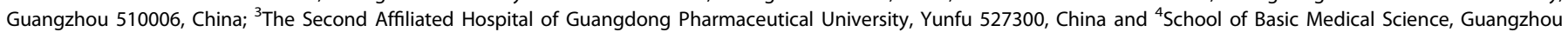
University of Chinese Medicine, Guangzhou 510006, China

Correspondence: Xiang-liang Deng (dxl@gdpu.edu.cn) or Lian Zhou (zl@gzucm.edu.cn)
}

Received: 12 October 2019 Accepted: 27 March 2020

Published online: 26 May 2020 
CD14 regulates the LPS-induced activation of the TLR4/NF-KB pathway and is found on the surface of many (but not all) TLR4expressing cells [6], such as macrophages, dendritic cells and intestinal epithelial cells. CD14 was the first identified pattern recognition receptor (PRR) that binds directly to LPS and plays an important role in sensitizing cells to minute quantities of LPS by chaperoning LPS molecules to the TLR4-MD-2 signaling complex; additionally, CD14 controls the subsequent endocytosis of TLR4 into the endosomal network, which regulates type I interferon (IFN) expression [6-8]. In the present study, we investigated whether CD14 plays a role in baicalin-mediated inhibition of TLR4/ NF-KB pathway activation and the subsequent inflammatory response.

\section{MATERIALS AND METHODS}

Drugs and reagents

Baicalin (98.58\% purity) was obtained from Chengdu Mansite Biological Technology Co., Ltd. (Chengdu, China). LPS (from Escherichia coli (0111:B4) was purchased from Sigma-Aldrich (St. Louis, MO, USA). Dextran sulfate sodium salt (DSS) was purchased from MP Biomedicals (Los Angeles, CA, USA). Griess reagent kits and nuclear and cytoplasmic protein extraction kits were obtained from Beyotime Biotechnology Co., Ltd. (Shanghai, China). ELISA kits for IL-6 and TNF-a were purchased from Lianke Biological Technology Co., Ltd. (Hangzhou, China). Rabbit anti-IL6 for immunohistochemistry was purchased from Sangon Biotech (Shanghai, China) Co., Ltd. (Shanghai, China). Rabbit anti-MyD88 (D80F5), phospho-NF-KB p65 (Ser 536) and SignalStain Boost IHC detection reagent (HRP, rabbit) were purchased from CST (Boston, MA, USA). Rabbit anti-CD14 $(\mathrm{H}-4)$ for Western blot analysis was purchased from Santa Cruz Biotechnology (Los Angeles, CA, USA). Mouse anti-CD14 for immunohistochemistry was purchased from Chinaway Biosciences Co., Ltd. (Guangzhou, China). Anti-mouse CD14-FITC, MHC-II-APC, TLR4-PE, and F4/80PE-Cy7 antibodies were purchased from eBioscience (San Diego, CA, USA). Anti-human CD14-FITC was purchased from BD Biosciences (Franklin Lakes, CA, USA). All RT-qPCR reagents were obtained from Takara Biomedical Technology Co., Ltd. (Beijing, China). Fetal bovine serum was purchased from Life Technologies (Carlsbad, CA, USA).

Cell culture

RAW264.7 cells (murine macrophages) were purchased from Shanghai Institutes for Biological Sciences (Shanghai, China) and were originally obtained from American Type Culture Collection (Manassas, USA) as described in our previous study [9]. HT-29 and HepG2 cells were obtained from the Type Culture Collection of the Chinese Academy of Sciences (Shanghai, China). RAW264.7shCD14 cells (CD14 was knocked down in RAW264.7 cells via shRNA interference) were prepared by Cytobiotech Biotechnology Co., Ltd. (Guangzhou, China). These cells were cultured at $37^{\circ} \mathrm{C}$ with $5 \% \mathrm{CO}_{2}$ in RPMI-1640 or Dulbecco's Modified Eagle's Medium (DMEM) containing $10 \%$ fetal bovine serum, $100 \mu \mathrm{g} / \mathrm{mL}$ streptomycin and $100 \mathrm{U} / \mathrm{mL}$ penicillin.

Cell viability assay

Cell viability was measured as described previously with minor modifications [10]. Briefly, cells were seeded in 96-well plates $\left(5 \times 10^{5}\right.$ cells $\left./ \mathrm{mL}\right)$, cultured in medium for $4 \mathrm{~h}$, treated with different concentrations of baicalin solution $(6.25,12.5,25,50$, 100 , and $200 \mu \mathrm{M}$ ), and incubated for $24 \mathrm{~h}$. Then, MTT was added to each well, and the cells were incubated for another $4 \mathrm{~h}$. The supernatant was removed, and dimethylsulfoxide dissolved (DMSO) was added to each well. The optical density of each well at $490 \mathrm{~nm}$ and $570 \mathrm{~nm}$ was measured on a microplate reader (Thermo Fisher Scientific, Waltham, USA), and cell viability was calculated.
$\mathrm{NO}$ and cytokine assays

Cells were seeded in 96 -well plates $\left(5 \times 10^{5}\right.$ cells $\left./ \mathrm{mL}\right)$. In baicalin pretreatment studies, the cells were pretreated with baicalin or medium for $2 \mathrm{~h}$, and then LPS or medium was added to stimulate the cells for another $24 \mathrm{~h}$. In baicalin posttreatment studies, the cells were pretreated with LPS or medium for $2 \mathrm{~h}$, and then baicalin or medium was added and incubated for another $24 \mathrm{~h}$. Cells that were treated only with medium were used as negative controls (control group), and those treated only with LPS were used as positive controls (LPS group). The supernatant was collected to determine the concentrations of NO and IL- 6 with Griess reagent and ELISA kits, respectively, according to the manufacturer's protocols.

Flow cytometry

Cells were seeded in 24-well plates $\left(1 \times 10^{6}\right.$ cells $\left./ \mathrm{mL}\right)$ and treated with baicalin as described above. After the supernatant was removed, the cells were collected and stained with fluorescence-conjugated monoclonal antibodies according to the manufacturer's instructions. The cells were resuspended in $300 \mu \mathrm{L}$ PBS and detected on a FACSCanto ${ }^{\mathrm{TM}}$ II flow cytometer (BD Biosciences).

\section{Western blot analysis}

Western blotting was used to detect the expression of MyD88, phospho-NF-KB p65, CD14 and TLR4. After pretreatment with baicalin for $2 \mathrm{~h}$, the cells were treated with LPS for $1-2 \mathrm{~h}$ and collected. Western blotting was performed according to previous studies $[11,12]$. Briefly, protein samples were prepared with a nuclear and cytoplasmic protein extraction kit. Protein concentrations were measured by the BCA protein assay method. The protein samples were separated by $10 \%$ SDSpolyacrylamide gel electrophoresis and transferred to polyvinylidene difluoride (PVDF) membranes (Millipore, Bedford, MA, USA). The membranes were blocked in $5 \%$ nonfat powdered milk or BSA at room temperature for $1 \mathrm{~h}$ and then incubated with primary antibodies at $4{ }^{\circ} \mathrm{C}$ overnight. After 3 washes in TBST, the membranes were incubated with horseradish peroxidase-conjugated anti-rabbit or anti-mouse lgG. Finally, the protein bands were visualized by ECL reagent and analyzed using ImageJ software.

\section{RT-qPCR}

RT-qPCR was used to determine the mRNA level of CD14 in the cells. Total cellular RNA was purified using the RNAiso Plus Kit. cDNA was synthesized using a Prime Script ${ }^{\mathrm{TM}} \mathrm{RT}$ Reagent Kit with gDNA Eraser. RT-qPCR was performed in a CFX Connect ${ }^{\mathrm{TM}}$ RealTime PCR Detection System using SYBR ${ }^{\circledR}$ Premix Ex Taq $^{\text {TM }}$ according to the manufacturer's instructions. The primer sequences are listed in Table 1. The relative expression of CD14 mRNA to GAPDH mRNA was determined using the ${ }^{\triangle \Delta} \mathrm{Ct}$ method.

Real-time dynamic live cell imaging assay

After digestion, the cell suspension was incubated with CD14-FITC antibody for $30 \mathrm{~min}$, washed, centrifuged and seeded in 96-well plates $\left(5 \times 10^{5}\right.$ cells $\left./ \mathrm{mL}\right)$. Finally, the plates were placed in a realtime dynamic live cell imaging system with a $\mathrm{CO}_{2}$ incubator, and photographs were taken to observe the intensity of green light (CD14-FITC).

\begin{tabular}{|lll|}
\hline Table 1. & Primers used for real-time RT-PCR. \\
\hline Gene & $\begin{array}{l}\text { Forward Primer sequence } \\
\left(5^{\prime}-3^{\prime}\right)\end{array}$ & $\begin{array}{l}\text { Reverse Primer sequence } \\
\left(5^{\prime}-3^{\prime}\right)\end{array}$ \\
\hline CD14 & TGGGCGAGAAAGGACTGAT & AGGAGCAAAGCCAAAGTTCC \\
GAPDH & ACCACAGTCCATGCCATCAC & TCCACCACCCTGTTGCTGTA \\
\hline
\end{tabular}


a

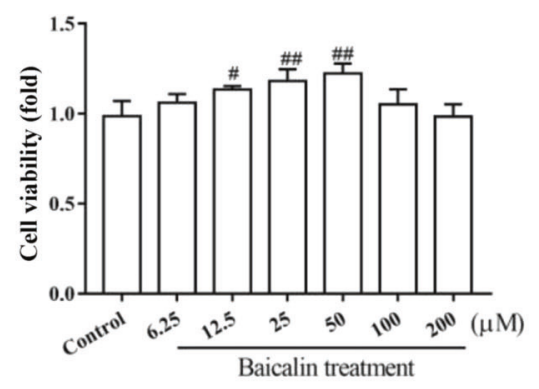

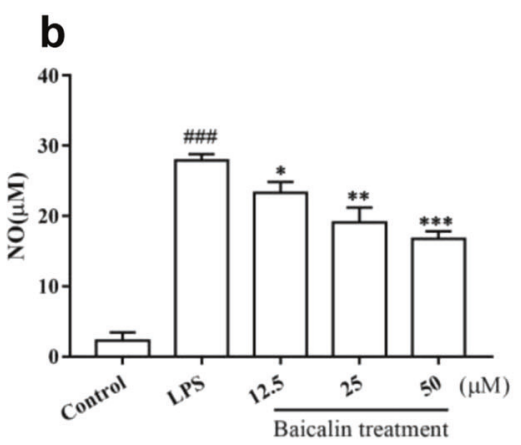

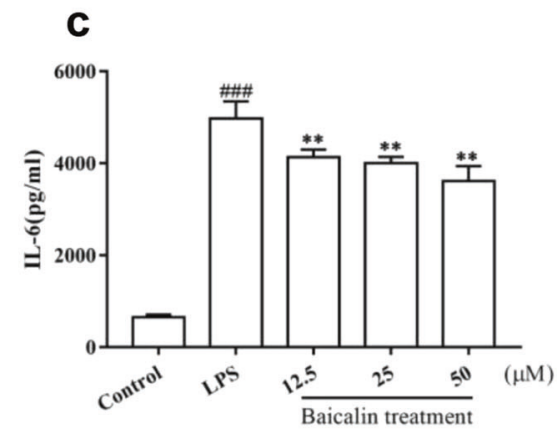

d

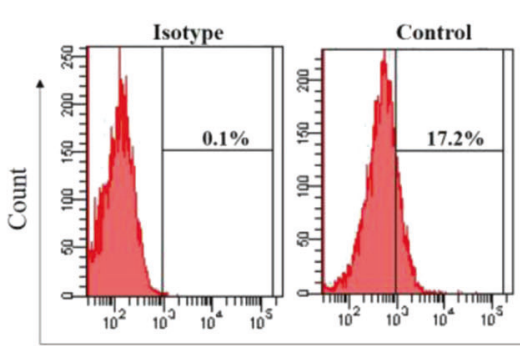

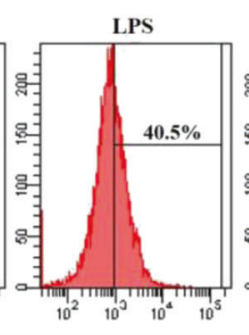

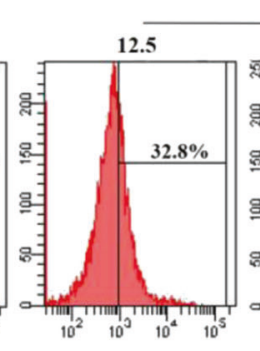

Baicalin treatment $(\mu \mathrm{M})$

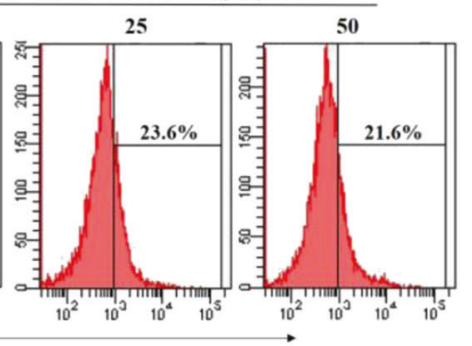

MHC-II-APC

e

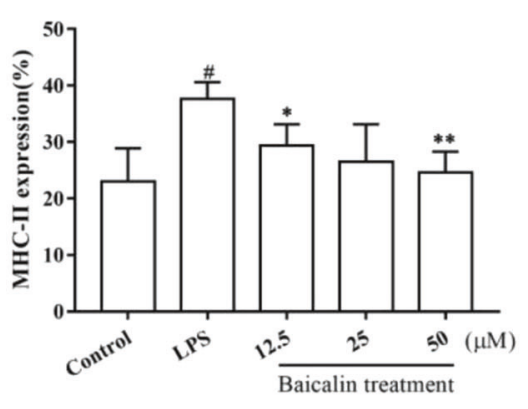

f

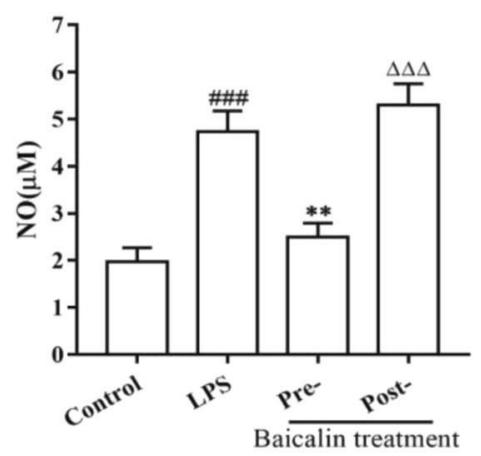

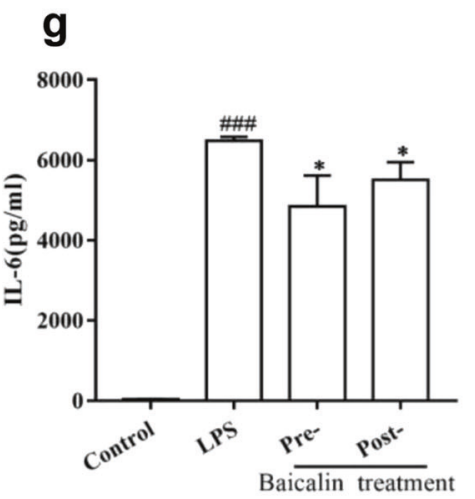
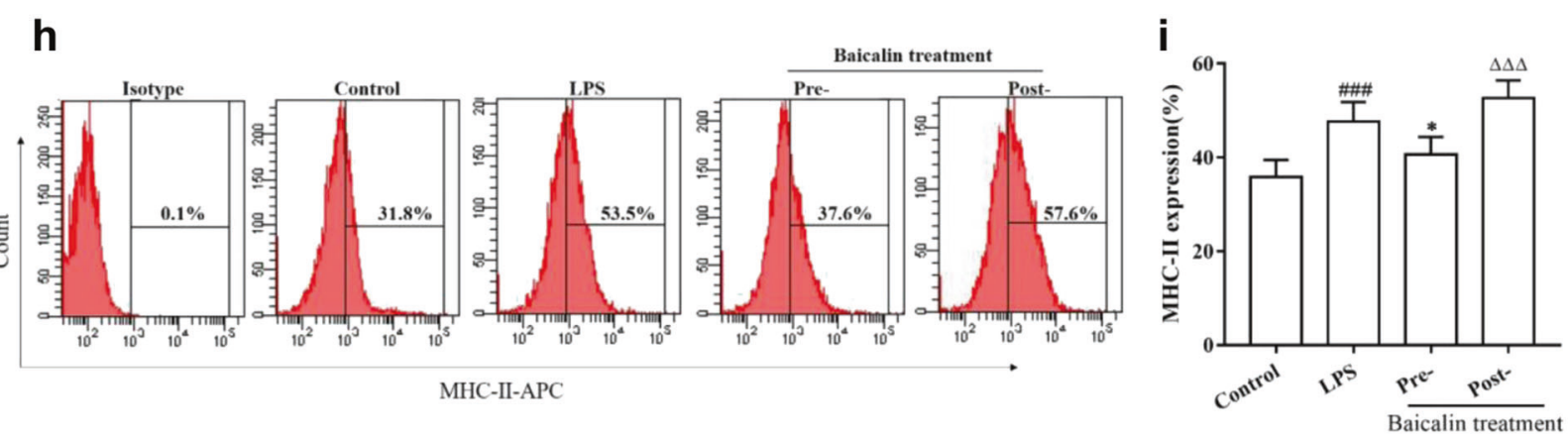

Fig. 1 Effects of baicalin pretreatment and posttreatment on LPS-induced inflammatory responses in RAW264.7 cells. a The cytotoxicity of baicalin on RAW264.7 cells by MTT assay. b, $\mathbf{c}$ The effects of baicalin pretreatment on the production of NO and IL- 6 in LPS-induced RAW264.7 cells. $\mathbf{d}$, e The effects of baicalin pretreatment on the expression of MHC II was measured by flow cytometry in LPS-induced RAW264.7 cells. $\mathbf{f}-\mathbf{i}$ The effects of baicalin posttreatment on the production of NO and IL-6 and the expression of MHC II in LPS-induced RAW264.7 cells; baicalin pretreatment was used as a positive control. Values are expressed as the means $\pm \mathrm{SD}, n=3$ in each group. ${ }^{\#} P<0.05,{ }^{\# \#} P<0.01$, and ${ }^{\# \# \#} P<0.001$ vs. the control group. ${ }^{*} P<0.05$, ${ }^{* *} P<0.01$, and ${ }^{* * *} P<0.001$ vs. the LPS group. ${ }^{\Delta \Delta \Delta} P<0.001$ vs. the pretreatment group. Baicalin was dissolved in $0.25 \%$ DMSO. RAW264.7 cells that were treated with medium were used as the control group.

Animals and treatments

Male Balb/c (20-22 g) and C57BL/6 mice were purchased from Guangdong Medical Laboratory Animal Center (Guangdong, China). The mice were maintained under standard temperature $\left(20 \pm 2^{\circ} \mathrm{C}\right)$, humidity $(55 \% \pm 2 \%)$ and $12 \mathrm{~h}$ light/dark cycle conditions with free access to food and water. The mice were allowed to acclimate to these conditions for at least 7 days before starting experiments. The experiments were approved by the Institutional Animal Care and Use Committee of Guangzhou University of Chinese Medicine, Guangzhou, China. All animal 
a

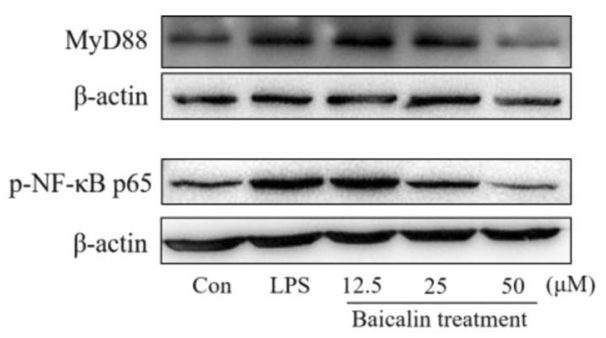

d

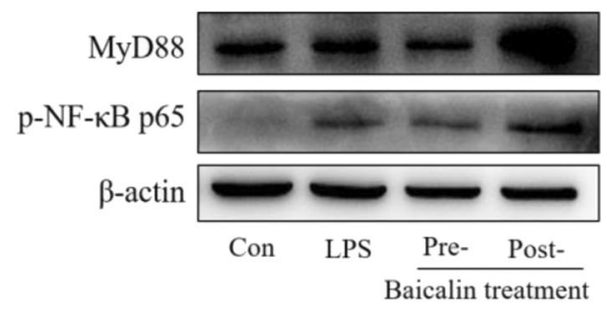

b

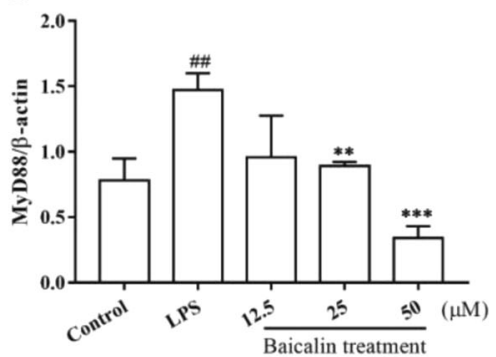

e

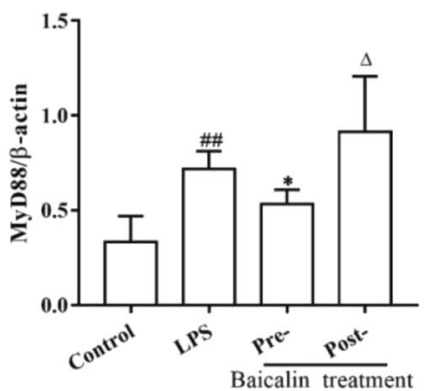

C

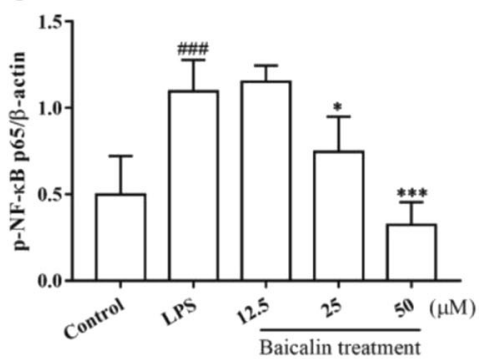

f

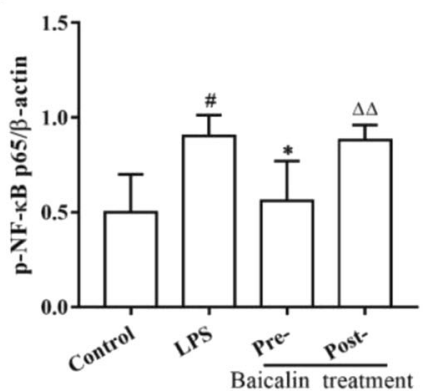

Fig. 2 Effect of baicalin pretreatment and posttreatment on the MyD88/NF-kB p65 pathway in LPS-induced RAW264.7 cells. The protein expression of MyD88 and p-NF-kB p65 was determined by Western blotting. a-c The effects of baicalin pretreatment on the protein expression of MyD88 and p-NF-kB p65 in the cytoplasm of LPS-induced RAW264.7 cells. d-f The effects of baicalin posttreatment on the protein expression of MyD88 and p-NF-KB p65 in the cytoplasm of LPS-induced RAW264.7 cells; baicalin pretreatment was used as a positive control. Values are expressed as the means \pm SD, $n=3$ in each group. ${ }^{\#} P<0.05,{ }^{\# \#} P<0.01$, and ${ }^{\# \# \#} P<0.001$ vs. the control group. ${ }^{*} P<0.05$, ${ }^{* *} P<0.01$, and ${ }^{* *} P<0.001$ vs. the LPS group. ${ }^{\Delta} P<0.05,{ }^{\Delta \Delta} P<0.01$ vs. the pretreatment group. Baicalin was dissolved in $0.25 \%$ DMSO. RAW264.7 cells that were treated with medium were used as the control group.

treatments were strictly in accordance with International Ethics Guidelines and the National Institutes of Health Guidelines Concerning the Care and Use of Laboratory Animals.

$\mathrm{BALB} / \mathrm{c}$ mice were randomly divided into 3 groups: control, LPS and LPS plus baicalin. The mice were pretreated orally with water (control and LPS group) or baicalin (LPS plus baicalin group, $100 \mathrm{mg} / \mathrm{kg}$ ) for 3 days before intraperitoneal injection of LPS (150 $\mu \mathrm{g} / \mathrm{kg}$, except for the control mice). After that, the weight and behavior of the mice were examined every $2 \mathrm{~h}$ for $24 \mathrm{~h}$.

C57BL/6 mice were randomly divided into 3 groups: control group, DSS group and DSS plus baicalin group. Mice in the DSS and DSS plus baicalin groups were given $3 \%$ DSS solution to drink freely for 5 days. On day 6 , mice in the DSS plus baicalin group were orally administered baicalin $(100 \mathrm{mg} / \mathrm{kg})$ every day for 5 days, while the other mice were given water. On day 11 , the mice were sacrificed for histological and immunohistochemical examination.

Histological and immunohistochemical examination The histological and immunohistochemical examinations were performed as described in our previous study [11] Briefly, the colons of the mice were fixed with $4 \%$ paraformaldehyde for $24 \mathrm{~h}$, dehydrated, and embedded in paraffin. Tissues were cut into $4 \mu \mathrm{m}$ sections and stained with hematoxylin and eosin (H\&E) for histopathological examination. For immunohistochemistry, the paraffin sections were baked at $60^{\circ} \mathrm{C}$ and then dipped successively in TO, a series of concentrations of ethyl alcohol and ultrapure water to remove the paraffin. Then, the paraffin sections were placed in a microwave oven for antigen unmasking and subsequently treated with $3 \%$ hydrogen peroxide to inhibit endogenous enzyme and blocking solution to block the nonspecific antigen. Then, the sections were incubated with anti-CD14 antibody or anti-IL- 6 antibody at $4{ }^{\circ} \mathrm{C}$ overnight, followed by incubation with secondary antibody for $1 \mathrm{~h}$. After the sections were reacted with DAB working solution, the tissues were observed under a microscope at a magnification of $\times 200$.

Statistical analysis

All data are presented as the mean \pm SD. The results were analyzed using GraphPad Prism software v5.0, and significant differences were determined by ANOVA or Student's $t$ test $(P<$ 0.05) where appropriate.

\section{RESULTS}

Baicalin pretreatment but not posttreatment prevents LPSinduced inflammatory responses in RAW264.7 cells To determine the effect of baicalin on cell viability, RAW264.7 cells were treated with $0 \mu \mathrm{M}$ (as a control), $6.25 \mu \mathrm{M}, 12.5 \mu \mathrm{M}, 25 \mu \mathrm{M}, 50$ $\mu \mathrm{M}, 100 \mu \mathrm{M}$, and $200 \mu \mathrm{M}$ baicalin for $24 \mathrm{~h}$, and the MTT assay was used. The results showed that baicalin was not cytotoxic to RAW264.7 cells in the range of $0-200 \mu \mathrm{M}$ (Fig. 1a). A previous study [13] showed that baicalin had anti-inflammatory activity in LPS-induced RAW264.7 cells. In this study, we further investigated whether baicalin prevented or inhibited LPS-induced inflammation in RAW264.7 cells.

To investigate the preventive effect of baicalin on LPS-induced inflammation, RAW264.7 cells were pretreated with $0 \mu \mathrm{M}$ (as a control), $12.5 \mu \mathrm{M}, 25 \mu \mathrm{M}$, and $50 \mu \mathrm{M}$ baicalin for $2 \mathrm{~h}$ and then exposed to LPS for another $24 \mathrm{~h}$. Flow cytometry analysis showed that pretreatment with baicalin prevented the expression of $\mathrm{MHC}$ II molecules, as well as the secretion of NO and IL-6, in LPSinduced RAW264.7 cells (Fig. 1b-e).

To investigate the inhibitory effect of baicalin on LPS-induced inflammation, RAW264.7 cells were pretreated with LPS for $2 \mathrm{~h}$ and then exposed to baicalin $(50 \mu \mathrm{M})$ for $24 \mathrm{~h}$, and cells that were pretreated with baicalin were used as a control. The results revealed that posttreatment with baicalin showed little inhibitory 
a

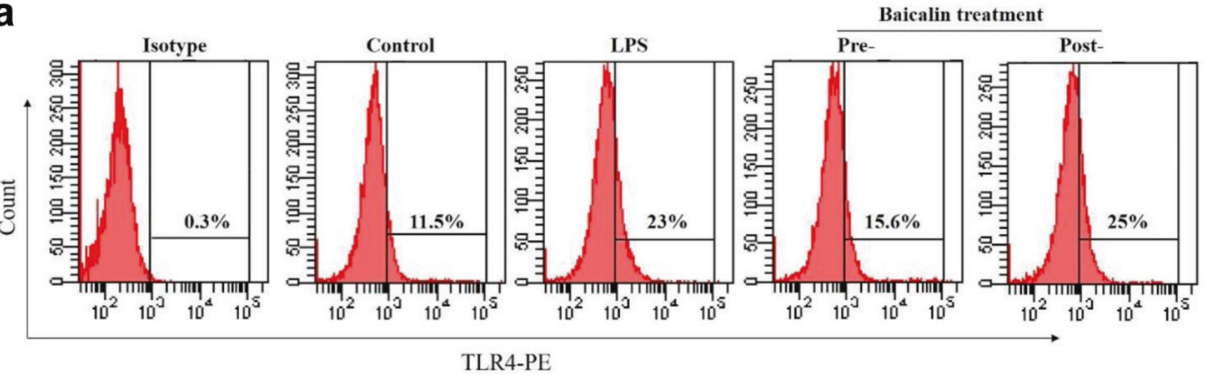

C

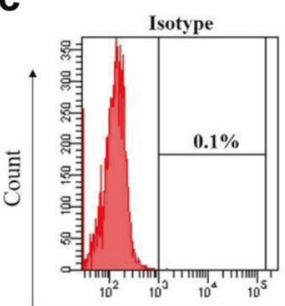

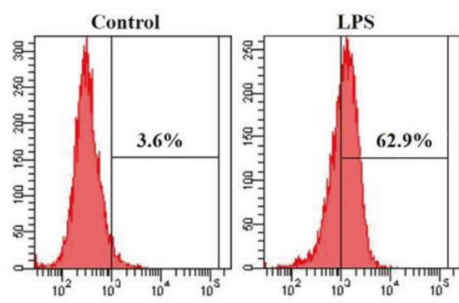

CD14-FITC

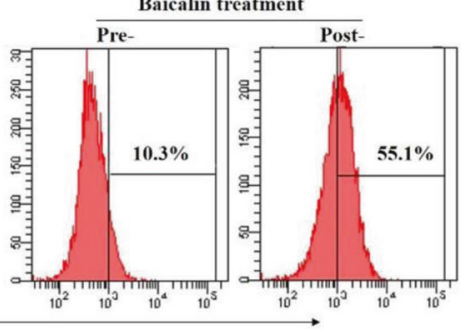

b

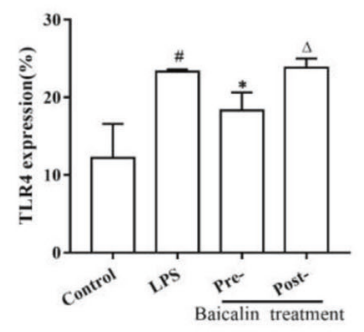

d

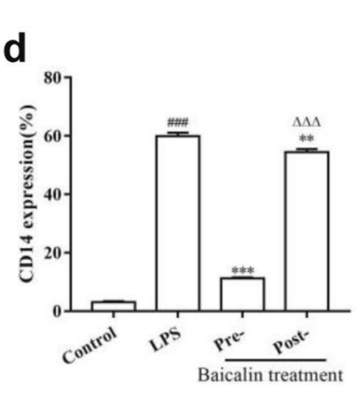

e

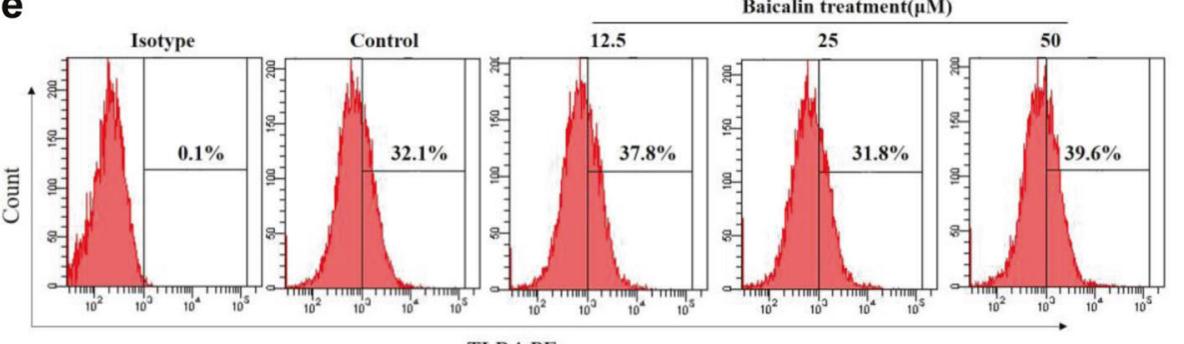

TLR4-PE

f

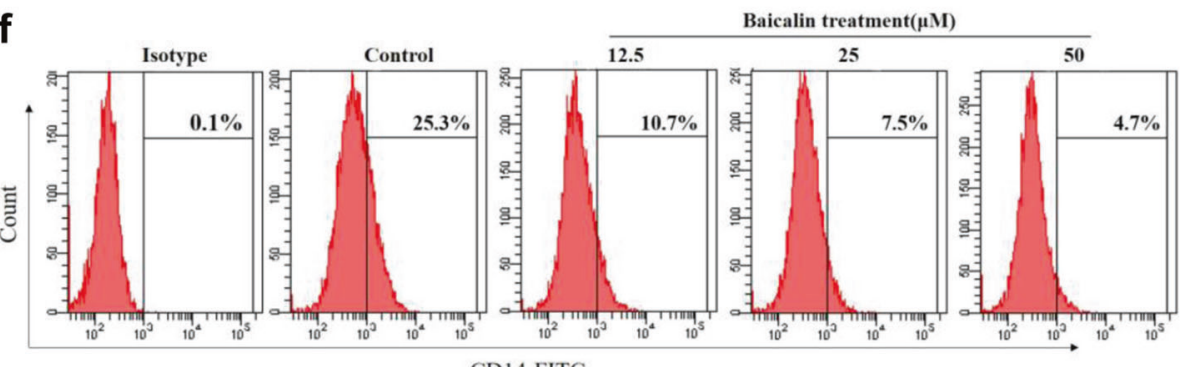

CD14-FITC
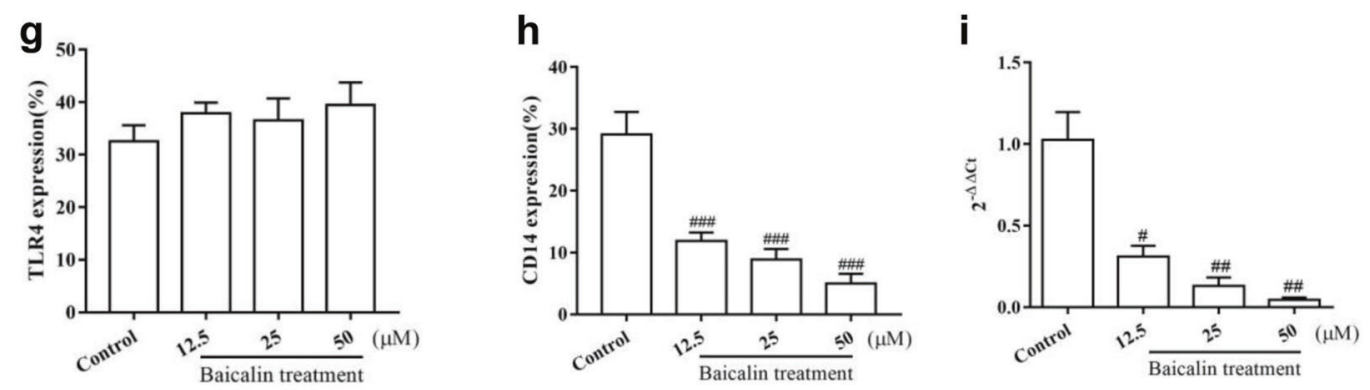

Fig. 3 Effects of baicalin treatment on CD14 and TLR4 expression in RAW264.7 cells. a-d The effects of pretreatment and posttreatment with baicalin $(50 \mu \mathrm{M})$ on the expression of CD14 and TLR4 in LPS-induced RAW264.7 cells was measured by flow cytometry. $\mathbf{e}-\mathbf{h}$ The effects of baicalin on the expression of CD14 and TLR4 in RAW264.7 cells without LPS stimulation was measured by flow cytometry. i The effects of baicalin on the mRNA expression of CD14 in RAW264.7 cells without LPS stimulation was measured by RT-PCR. Values are expressed as the means \pm SD, $n=3$ in each group. ${ }^{\#} P<0.05$, ${ }^{\# \#} P<0.01$, ${ }^{\# \# \#} P<0.001$ vs. the control group. ${ }^{*} P<0.05,{ }^{* *} P<0.01$, and ${ }^{* * *} P<0.001$ vs. the LPS group. ${ }^{\Delta} P<0.05,{ }^{\Delta \Delta \Delta} P<0.001$ vs. the pretreatment group. Baicalin was dissolved in $0.25 \%$ DMSO. RAW264.7 cells that were treated with medium were used as the control group. 
effect on the expression of MHC II molecules or the secretion of NO but not IL-6 (Fig. 1f-i) in LPS-induced RAW264.7 cells.

Baicalin pretreatment but not posttreatment decreases activation of the MyD88/NF-kB p65 signaling pathway in RAW264.7 cells LPS-induced inflammatory responses involve activation of the MyD88-dependent inflammatory signaling pathway, which in turn activates NF-kB p65 [14]. To investigate the effects of pretreatment or posttreatment with baicalin on activation of the MyD88/ NF-KB p65 signaling pathway, RAW264.7 cells were treated as previously described, and then the expression levels of MyD88 and $\mathrm{p}-\mathrm{NF}-\mathrm{KB}$ p65 in the cytoplasm were determined by Western blot (WB) analysis. WB analysis showed that pretreatment with baicalin decreased the expression levels of MyD88 and p-NF-kB p65 in the cytoplasm of LPS-induced RAW264.7 cells (Fig. 2a-c). As expected, posttreatment with baicalin showed little effect on the expression of the MHC II molecules, MyD88 or p-NF-kB p65 in the cytoplasm of LPS-induced RAW264.7 cells (Fig. $2 d-f$ ).

Baicalin prevents LPS-induced inflammatory responses by inhibiting CD14 expression in RAW264.7 cells

CD14 and TLR4 are PRRs on the cell membrane that recognize LPS during the activation of RAW264.7 cells. This is a classical process that is important in the study of anti-inflammatory mechanisms. To investigate the mechanisms by which baicalin pretreatment decreased activation of the NF-KB p65 signaling pathway and the subsequent inflammatory response, CD14 and TLR4 expression levels were determined after RAW264.7 cells were treated as previously described. The flow cytometry results revealed that pretreatment but not posttreatment with baicalin had significant effects on the expression of CD14 and TLR4 in LPS-induced RAW264.7 cells (Fig. 3a-d). We hypothesized that baicalin pretreatment directly inhibits CD14 or TLR4 expression and then prevent LPS from binding to RAW264.7 cells. To prove our hypothesis, we treated RAW264.7 cells with baicalin for $24 \mathrm{~h}$ and determined the expression of CD14 and TLR4. The flow cytometry results showed that baicalin directly inhibited the expression of CD14 but not TLR4 on the surface of RAW264.7 cells (Fig. 3e-h). The RT-PCR results confirmed that baicalin inhibited the mRNA expression of CD14 (Fig. 3i).

Previous studies reported that CD14 plays an important role in sensitizing the cells to minute quantities of LPS by chaperoning LPS molecules to the TLR4-MD-2 signaling complex, while a high concentration of LPS directly binds to TLR4-MD-2 $[7,8]$. Our results indicated that baicalin pretreatment decreased activation of the NF-KB p65 signaling pathway by inhibiting CD14 expression in LPS-induced RAW264.7 cells. To confirm this hypothesis, we silenced CD14 in RAW264.7 cells by shRNA transfection (RAW264.7 cells ${ }^{\text {shCD14 }}$, Supplementary Fig. S1), which had little effect on TLR4 expression (Fig. $4 a-c$ ). Then, these cells were pretreated with baicalin for $2 \mathrm{~h}$ and then exposed to a high dose of LPS and baicalin for another $24 \mathrm{~h}$. The results revealed that pretreatment with baicalin did not decrease the secretion of IL-6 or NO (Fig. 4d, e) or the expression of p-NF-KB p65 in RAW264.7 cells shCD14 (Fig. 4f, g). Thus, baicalin pretreatment prevented LPS-induced inflammation in RAW264.7 cells in a CD14-dependent manner.

Baicalin also inhibits the expression of CD14 and prevents LPSinduced activation of the MyD88/NF-KB p65 pathway in HT-29 and HepG2 cells

To investigate whether baicalin also inhibits CD14 expression and prevents LPS-induced activation of the MyD88/NF-KB p65 pathway in other cells, HT-29 and HepG2 cells were used because studies have reported that they express CD14 $[15,16]$. Consistent with the above methods, we continued to use LPS stimulation to establish an inflammatory model to verify the anti-inflammatory effects of baicalin and its inhibitory effect on CD14. First, the MTT assay was used to detect the toxicity of baicalin (at doses of 12.5 a
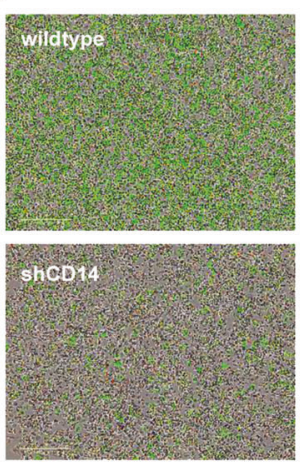

b
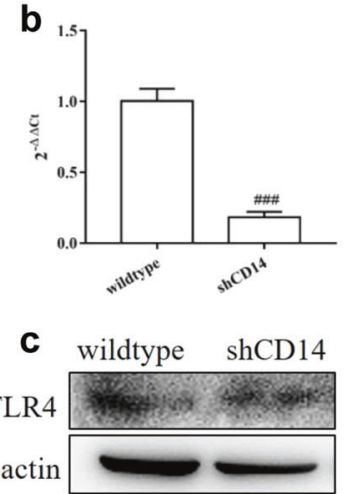

d

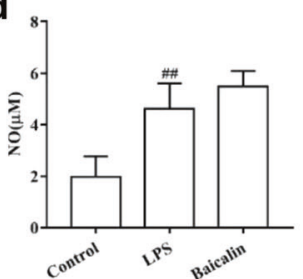

e

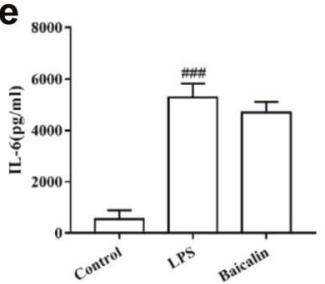

f

g
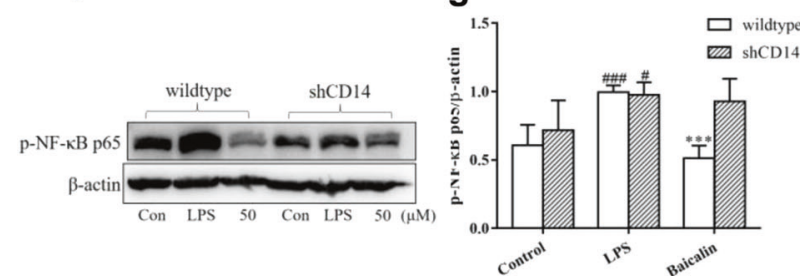

Fig. 4 Effects of baicalin on inflammatory responses in RAW264.7 cells with CD14 knockdown. a-c RAW264.7 cells with CD14 knockdown were prepared by shRNA transfection (shCD14). The expression of CD14 and TLR4 in RAW264.7 cells with CD14 knockdown by real-time dynamic live cell imaging system, PCR and Western blotting, respectively. Wild-type cells were used as a control. d, e The effects of baicalin pretreatment $(50 \mu \mathrm{M})$ on the production of NO and IL-6 in LPS-induced RAW264.7 cells with CD14 knockdown. $\mathbf{f}, \mathbf{g}$ The effects of baicalin pretreatment $(50 \mu \mathrm{M})$ on the protein expression of p-NF-кB p65 in the cytoplasm of LPS-induced RAW264.7 cells with CD14 knockdown and wild-type cells. Values are expressed as the means $\pm S D, n=3$ in each group. ${ }^{\#} P<0.05$,

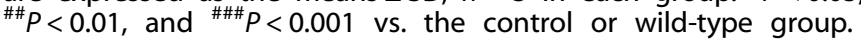
***P $<0.001$ vs. the LPS group. Baicalin was dissolved in $0.25 \%$ DMSO. Cells that were treated with medium were used as the control group.

$\mu \mathrm{M}, 25 \mu \mathrm{M}$, and $50 \mu \mathrm{M})$ to the cells. We found that baicalin was not toxic to the cells (Supplementary Fig. S2). Next, we used flow cytometry and WB analysis to determine the expression of CD14, MyD88, and p-NF-kB p65 in LPS-induced HT-29 and HepG2 cells. The results revealed that pretreatment with baicalin also decreased the expression of CD14, MyD88, and p-NF-KB p65 in HT-29 (Fig. 5a-d) and HepG2 cells (Fig. 5e-i).

Baicalin inhibits the expression of CD14 in tissues and exerts protective effects against LPS-induced inflammation in mice The in vitro experiments demonstrated that baicalin exhibits an anti-inflammatory effect by inhibiting the expression of CD14 and preventing the activation of the NF-KB p65 pathway in LPSinduced RAW264.7 cells, HT-29 cells and HepG2 cells. This indicated that the inhibitory effect of baicalin on CD14 expression might be used to treat LPS-induced inflammatory diseases in vivo. To confirm this hypothesis, we established two inflammatory models in mice for in vivo experiments. 

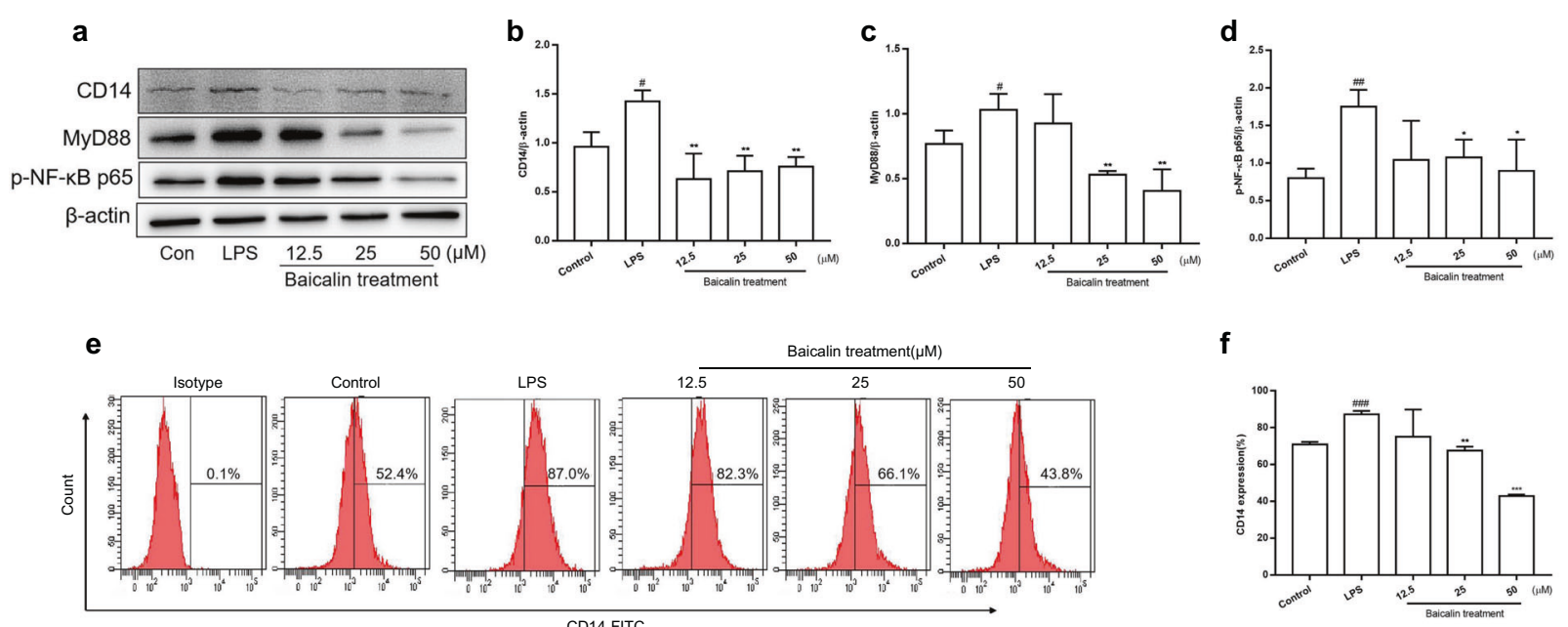

f

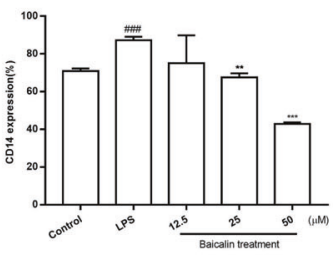

g

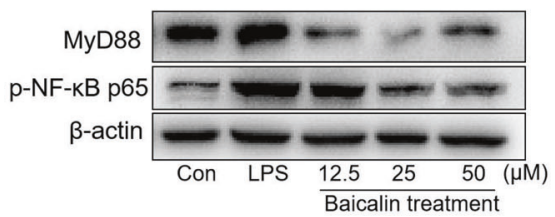

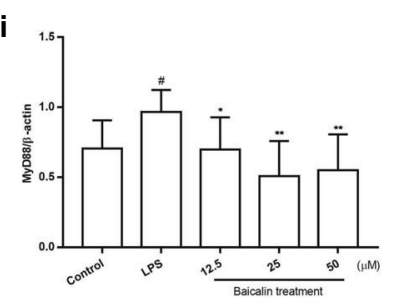

Fig. 5 Effects of baicalin pretreatment on CD14 and the MyD88/NF-KB p65 pathways in HT-29 and HepG2 cells. a-d Effects of baicalin pretreatment on CD14 and the MyD88/NF-KB p65 pathways in HT-29 cells. Western blotting was used to measure the protein expression of

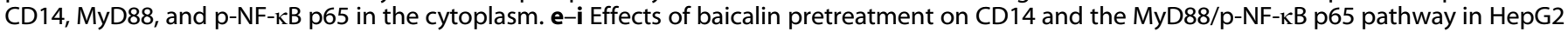
cells. Flow cytometry was used to measure CD14 expression. Western blotting was used to measure the protein expression of MyD88 and p-

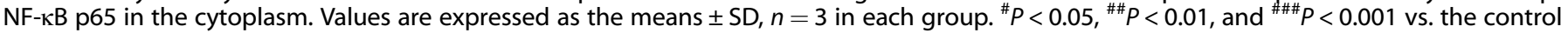
group. ${ }^{*} P<0.05$, ${ }^{* *} P<0.01$, and ${ }^{* * *} P<0.001$ vs. the LPS group. Baicalin was dissolved in $0.25 \%$ DMSO. The cells that were treated with medium were used as the control group.

In one study, Balb/c mice were pretreated with baicalin orally for 3 days and then injected intraperitoneally with LPS, except for the control group. As shown in Fig. $6 \mathrm{a}$, at $12 \mathrm{~h}$ after LPS injection, the body weights of the mice in the LPS group continued to decrease for up to $24 \mathrm{~h}$, while those in the baicalin pretreatment group began to recover at $16 \mathrm{~h}$ after LPS injection and showed a significant recovery at $24 \mathrm{~h}$. During this period, there was significant diarrhea in the LPS group, while mice in the baicalin group had significantly less severe diarrhea. Regarding the lymphoid organ index, compared with the control group, the LPS group showed a significant increase in spleen and thymus indexes. Compared with the LPS group, the baicalin group showed a significant decrease in the lymphoid organ indexes (Fig. 6b, c). The immunohistochemical analysis results revealed that the expression of CD14 and IL- 6 were both increased significantly in mouse colon tissue in the LPS group, while those in the baicalin group were significantly decreased compared with the LPS group (Fig. 6d).

In another study, C57BL/6 mice were given 3\% DSS solution to drink freely for 5 days to establish the UC model, except for the control mice. On day 6 , mice in the treatment group were orally administered baicalin solution $(100 \mathrm{mg} / \mathrm{kg})$ or mesalazine every day for 5 days, while the other mice were given water. The results revealed that baicalin treatment restored colon length and spleen index nearly to normal levels (Fig. 6e-g), alleviated the severity of ulcers and reduced the expression of CD14 and IL- 6 in the colonic mucosa compared with those in the DSS group (Fig. 6h, Supplementary Fig. S3).

\section{DISCUSSION}

LPS plays an important role in some inflammatory diseases, such as inflammatory bowel disease, sepsis and septic shock. In this study, we investigated how baicalin prevents LPS-induced activation of the TLR4/NF-KB p65 pathway and subsequent inflammation in vitro and in vivo. Our results showed that baicalin prevents LPS-induced activation of the TLR4/NF-KB p65 pathway by inhibiting the expression of CD14 molecules.

First, we investigated the preventive and inhibitory effects of baicalin on LPS-induced inflammation. Although previous studies have demonstrated that baicalin has anti-inflammatory activity by inhibiting activation of the NF-KB p65 pathway $[12,13,17-19]$, the detailed mechanism is unknown. In the present study, we found that baicalin pretreatment but not posttreatment prevented LPSinduced inflammatory responses, such as the expression of $\mathrm{MHC}$ II molecules and the secretion of the inflammatory mediator $\mathrm{NO}$ in RAW264.7 cells. LPS induces activation of the MyD88/NF-KB p65 pathway and promotes the release of inflammatory cytokines [1]. Our results showed that baicalin pretreatment but not posttreatment decreased activation of the MyD88/NF-KB p65 pathway in LPS-induced RAW264.7 cells. This nicely illuminates why baicalin pretreatment but not posttreatment prevents LPS-induced inflammatory responses. Our results demonstrate that baicalin has a preventive not an inhibitory effect on LPS-induced inflammation.

Second, we further investigated how baicalin decreased LPSinduced activation of the MyD88/NF-KB p65 pathway. CD14 and TLR4 are the first PRRs on the cell that bind to LPS; more precisely, CD14 transfers minute quantities of LPS to TLR4 and controls the subsequent endocytosis of TLR4 into the endosomal network [6]. In other words, CD14 and TLR4 are upstream molecules through which LPS induces activation of the MyD88/NF-KB p65 pathway. We found that baicalin pretreatment but not posttreatment decreased the expression of CD14 and TLR4 in LPS-induced RAW264.7 cells. Because CD14 is the first PRR, directly binds to 
a

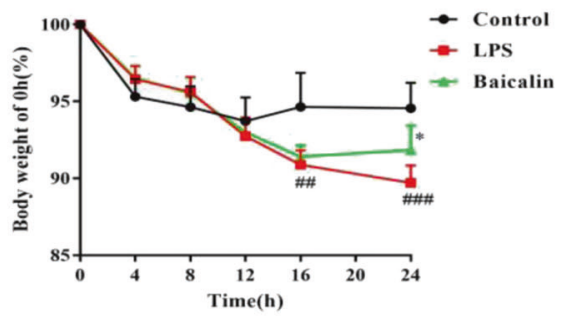

b

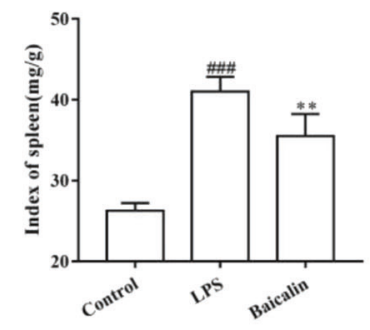

C

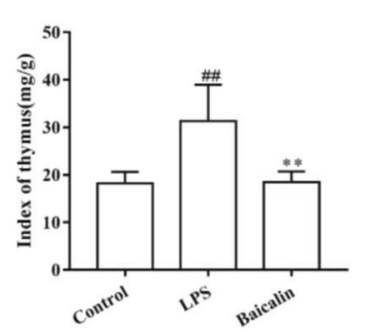

e

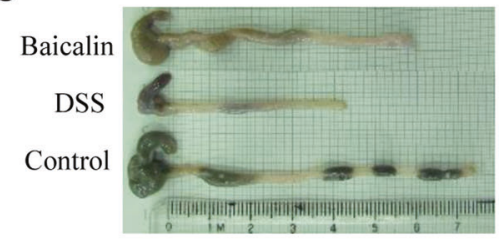

f

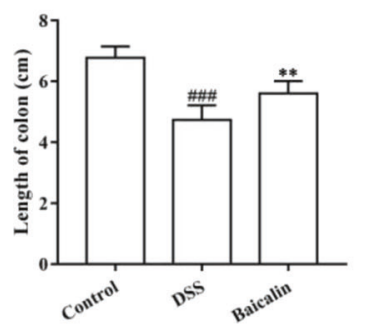

g

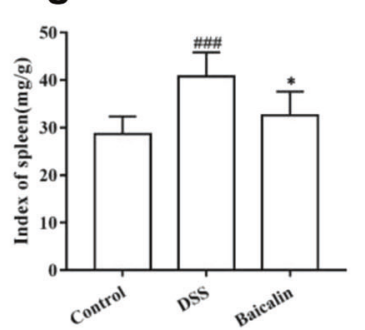

d

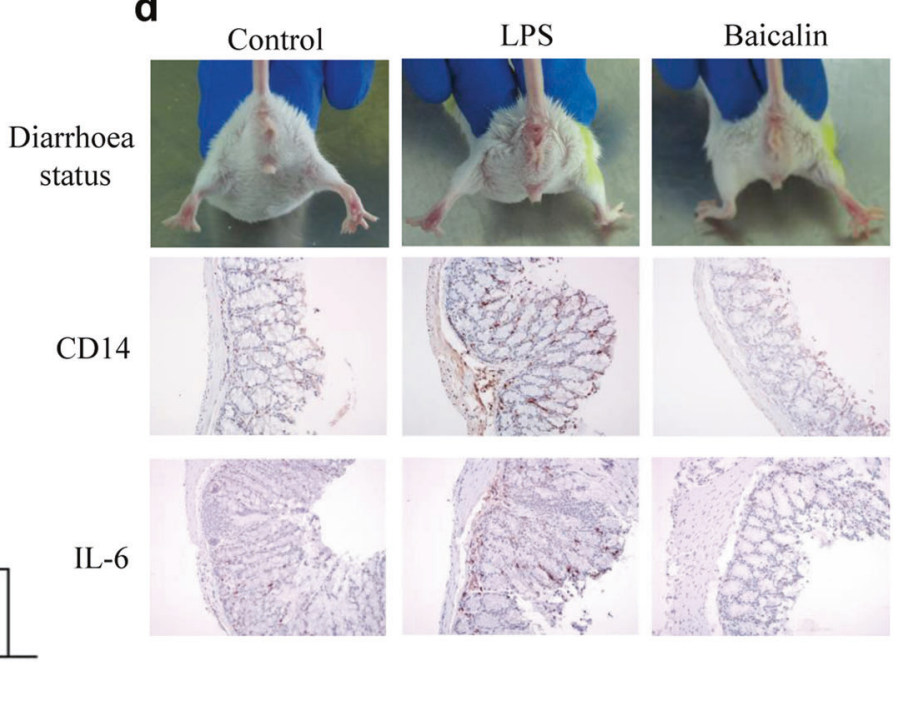

h

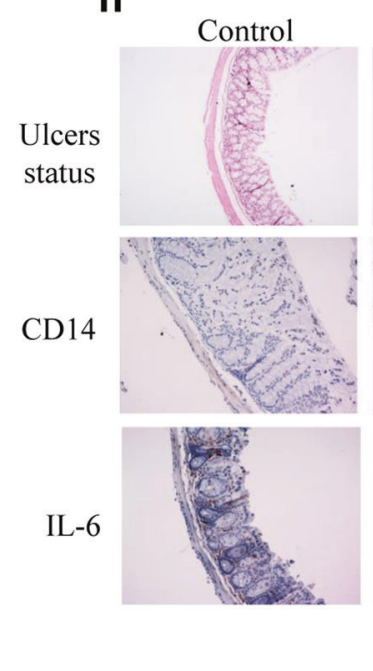

LPS

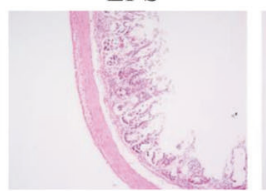

Baicalin

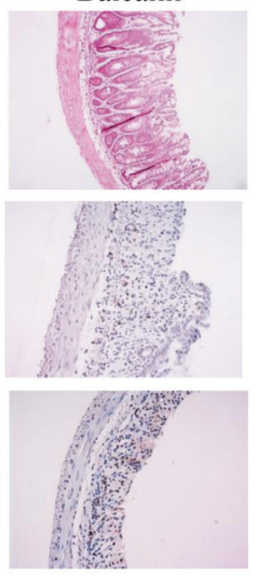

Fig. 6 Effects of baicalin treatment on LPS-induced inflammation in mice. a-d Effects of baicalin treatment on inflammation in BALB/c mice with intraperitoneal injection of LPS. Except for mice in the control group, mice in the LPS group and baicalin group were intraperitoneally injected with LPS $(150 \mu \mathrm{g} / \mathrm{kg})$. The body weight and spleen and thymus index were investigated. The secretion of CD14 and IL- 6 in colon tissue was measured by immunocytochemistry. $\mathbf{e}-\mathbf{h}$ Effects of baicalin treatment on inflammation in C57BL/ 6 mice. The mice were induced with DSS to establish the UC model. The effects of baicalin on the colon length and spleen index were investigated. The ulcer status was investigated by H\&E staining. The secretion of CD14 and IL- 6 in colon tissue was measured by immunocytochemistry. Values are expressed as the means \pm SD, $n=6$ in each group. ${ }^{\# \#} P<0.01$ and ${ }^{\# \# \#} P<0.001$ vs. the control group. ${ }^{*} P<0.05$ and ${ }^{* *} P<0.01$ vs. the LPS or DSS group.

minute quantities of LPS and transfers LPS to TLR4, we hypothesized that baicalin prevented LPS-induced activation of the MyD88/NF-KB p65 pathway by inhibiting CD14 expression. To verify this hypothesis, we further investigated the effects of baicalin on the expression of CD14 and TLR4 in RAW264.7 cells. Our results showed that baicalin directly inhibited the protein and mRNA expression of CD14 but not TLR4 in RAW264.7 cells. Another piece of strong evidence in our study to support the conclusion is that in RAW264.7 cells with CD14 knockdown, baicalin did not inhibit inflammatory responses or activation of the MyD88/NF-KB p65 pathway in the presence of high concentrations of LPS. This is consistent with the fact that when concentrations are high, LPS directly binds to TLR4 in a CD14-independent manner and activates the MyD88/NF-KB p65 pathway [20]. Our results are also consistent with a previous study showing that siRNA targeting mCD14 inhibits inflammation in LPS-stimulated RAW264.7 cells [21]. Thus, our results demonstrate that baicalin prevents LPS-induced activation of the MyD88/NF-KB p65 pathway by inhibiting CD14 expression in RAW264.7 cells.

Next, we continued to explore whether this mechanism exists in other cell lines and in vivo. CD14 is found on the surface of not only macrophages but also many (but not all) TLR4-expressing cells, such as liver cells and intestinal epithelial cells $[15,16]$. LPS binds to these cells and induces activation of the MyD88/NF-KB p65 pathway and the subsequent inflammatory responses, which play an important role in the occurrence and development of related inflammatory diseases [22]. Thus, baicalin might also prevent LPS-induced activation of the MyD88/NF-KB p65 pathway by inhibiting CD14 expression in these cells. As expected, baicalin inhibited the expression of CD14 and activation of the MyD88/NFKB p65 pathway in LPS-induced hepatocyte-derived HepG2 cells and intestinal epithelial-derived HT-29 cells. Consistent with the in vitro studies, baicalin treatment had a protective effect by inhibiting CD14 expression and inflammation in mice that were 
intraperitoneally injected with LPS and in DSS-induced UC mice. Intraperitoneal injection of LPS is used to establish a model of intestinal injury, which activates the intestinal inflammatory response $[23,24]$. CD14 has been identified as an IBD candidate gene that is mainly distributed in the distal colon and cecum of mice [25] CD14 is only expressed at a low level in the uninflamed intestine and is upregulated after DSS treatment [26-28]. A previous study reported the therapeutic effect of baicalin on ulcerative colitis by reducing the expression of TLR4 in the colon $[4,5,29]$. In the present study, taken together with our in vitro and in vivo results, these therapeutic effects of baicalin possibly work by inhibiting CD14 expression.

In conclusion, we found that baicalin prevented LPS-induced activation of the TLR4/NF-KB p65 pathway and inflammation by inhibiting the expression of CD14. These findings demonstrate that CD14 is an important target for preventing LPS-induced inflammation. Furthermore, these findings also promote the therapeutic utilization of baicalin in preventing LPS-induced inflammatory disease progression.

\section{ACKNOWLEDGEMENTS}

This work was supported by the National Natural Science Foundation of China [Grant numbers 81703785, 81673668]; the Characteristic Innovation Projects of Colleges and Universities in Guangdong Province, China [Grant number 2017KTSCX042]; and the Natural Science Foundation of Guangdong Province [Grant number 2017A030313657].

\section{AUTHOR CONTRIBUTIONS}

$X L D$ and $L Z$ contributed to the conception and design of the study. YJF, XL, BX, SWH, $\mathrm{CL}, \mathrm{QW}, \mathrm{SL}$, and JYC contributed to the acquisition of data. YJF and $\mathrm{XL}$ analyzed the data. YJF and XLD wrote the manuscript. XLD, LZ, and XL contributed to the material and technological support. All authors read and approved the manuscript.

\section{ADDITIONAL INFORMATION}

The online version of this article (https://doi.org/10.1038/s41401-020-0411-9) contains supplementary material, which is available to authorized users.

Conflict of interest: The authors declare that they have no conflict of interest

\section{REFERENCES}

1. Medzhitov R. Origin and physiological roles of inflammation. Nature. 2008;454:428-35.

2. Dinda B, Dinda S, DasSharma S, Banik R, Chakraborty A, Dinda M. Therapeutic potentials of baicalin and its aglycone, baicalein against inflammatory disorders. Eur J Med Chem. 2017;131:68-80.

3. Doyle SL, O'Neill LAJ. Toll-like receptors: from the discovery of NF-kappaB to new insights into transcriptional regulations in innate immunity. Biochem Pharmacol. 2006;72:1102-13.

4. Girardin SE, Boneca IG, Viala J, Chamaillard M, Labigne A, Thomas G, et al. Nod2 is a general sensor of peptidoglycan through muramyl dipeptide (MDP) detection. J Biol Chem. 2003;278:8869-72.

5. Yin XX, Krikorian P, Logan T, Csizmadia V. Induction of RIP-2 kinase by proinflammatory cytokines is mediated via NF-kappa B signaling pathways and involves a novel feed-forward regulatory mechanism. Mol Cell Biochem. 2010;333:251-9.

6. Zanoni I, Ostuni R, Marek LR, Barresi S, Barbalat R, Barton GM, et al. CD14 controls the LPS-induced endocytosis of toll-like receptor 4. Cell. 2011;147:868-80.

7. Wright SD, Ramos RA, Tobias PS, Ulevitch RJ, Mathison JC. CD14, a receptor for complexes of lipopolysaccharide (LPS) and LPS binding protein. Science. 1990;249:1431-33.
8. Moore KJ, Andersson LP, Ingalls RR, Monks BG, Li R, Arnaout MA, et al. Divergent response to LPS and bacteria in CD14-deficient murine macrophages. J Immunol. 2000;165:4272-80.

9. Deng XL, Liu Q, Fu YJ, Luo X, Hu MH, Ma FL, et al. Effects of Lycium barbarum polysaccharides with different molecular weights on function of RAW264.7 macrophages. Food Agr Immunol. 2018;29:808-20.

10. Deng XL, Li XL, Luo S, Zheng YY, Luo X, Zhou L. Antitumor activity of Lycium barbarum polysaccharides with different molecular weights: an in vitro and in vivo study. Food Nutr Res. 2017;61:1399770.

11. Luo S, Deng XL, Liu Q, Pan ZF, Zhao ZX, Zhou L, et al. Emodin ameliorates ulcerative colitis by the flagellin-TLR5 dependent pathway in mice (vol 59, pg 269, 2018). Int Immunopharmacol. 2018;62:337.

12. Ye L, Tao $\mathrm{YH}$, Wang YM, Feng $\mathrm{T}$, Li H. The effects of baicalin on the TLR2/ 4 signaling pathway in the peripheral blood mononuclear cells of a lipopolysaccharide-induced rat fever model. Int immunopharmacol. 2015;25:106-11.

13. Yun Y, Wang CZ, Gui L, Li ZX. Effect of baicalin on expression of TLR4 in RAW264.7 cells infected by ESBLs Escherichia coli. Zhongguo Zhong yao za zhi = Zhongguo zhongyao zazhi = China J Chin Mater Med. 2013;38:1590-4.

14. Flannery S, Bowie AG. The interleukin-1 receptor-associated kinases: critical regulators of innate immune signalling. Biochem Pharmacol. 2010;80:1981-91.

15. Su GL, Dorko K, Strom SC, Nussler AK, Wang SC. CD14 expression and production by human hepatocytes. J Hepatol. 1999;31:435-42.

16. Bas S, Gauthier BR, Spenato U, Stingelin S, Gabay C. CD14 is an acute-phase protein. J Immunol. 2004;172:4470-9.

17. Zhang CL, Zhang S, He WX, Lu JL, Xu YJ, Yang JY, et al. Baicalin may alleviate inflammatory infiltration in dextran sodium sulfate-induced chronic ulcerative colitis via inhibiting IL-33 expression. Life Sci. 2017;186:125-32.

18. Dong SJ, Zhong YQ, Lu WT, Li GH, Jiang HL, Mao B. Baicalin inhibits lipopolysaccharide-induced inflammation through signaling NF-kappaB pathway in HBE16 airway epithelial cells. Inflammation. 2015;38:1493-501.

19. Guo MY, Zhang NS, Li DP, Liang DJ, Liu ZC, Li FY, et al. Baicalin plays an antiinflammatory role through reducing nuclear factor-kappa $B$ and p38 phosphorylation in S. aureus-induced mastitis. Int Immunopharmacol. 2013;16:125-30.

20. Plociennikowska A, Hromada-Judycka A, Borzecka K, Kwiatkowska K. Cooperation of TLR4 and raft proteins in LPS-induced pro-inflammatory signaling. Cell Mol Life Sci. 2015;72:557-81.

21. Ming L. siRNA targeting mCD14 inhibits TNF-a, MIP-2, and IL-6 secretion and NO production from LPS-induced RAW264.7 cells. Appl Microbiol Biotechnol. 2011;92:115.

22. Garg AD, Kaczmarek A, Krysko O, Vandenabeele P, Krysko DV, Agostinis P. ER stress-induced inflammation: does it aid or impede disease progression? Trends Mol Med. 2012;18:589-98.

23. Wan Y, Fu YQ, Wang FL, Sinclair AJ, Li D. Protective effects of a lipid extract from Hard-Shelled Mussel (Mytilus coruscus) on intestinal integrity after lipopolysaccharide challenge in mice. Nutrients. 2018;10:860.

24. Cui Y, Wang Q, Sun R, Guo L, Wang M, Jia J, et al. Astragalus membranaceus (Fisch.) Bunge repairs intestinal mucosal injury induced by LPS in mice. BMC Complement Alter Med. 2018;18:230.

25. Buchheister S, Buettner M, Basic M, Noack A, Breves G, Buchen B, et al. CD14 plays a protective role in experimental Inflammatory bowel disease by enhancing intestinal barrier function. Am J Pathol. 2017;187:1106-20.

26. Ortega-Cava CF, Ishihara S, Rumi MA, Kawashima K, Ishimura N, Kazumori H, et al. Strategic compartmentalization of toll-like receptor 4 in the mouse gut. J Immunol. 2003;170:3977-85.

27. Kamada N, Hisamatsu T, Okamoto S, Chinen H, Kobayashi T, Sato T, et al. Unique $\mathrm{CD} 14$ intestinal macrophages contribute to the pathogenesis of Crohn disease via IL-23/IFN-gamma axis. J Clin Invest. 2008;118:2269-80.

28. Thiesen $S$, Janciauskiene $S$, Uronen-Hansson $H$, Agace $W$, Hogerkorp $C M$, Spee $P$, et al. CD14(hi)HLA-DR(dim) macrophages, with a resemblance to classical blood monocytes, dominate inflamed mucosa in Crohn's disease. J Leukoc Biol. 2014:95:531-41.

29. Guo S, Al-Sadi R, Said HM, Ma TY. Lipopolysaccharide causes an increase in intestinal tight junction permeability in vitro and in vivo by inducing enterocyte membrane expression and localization of TLR-4 and CD14. Am J Pathol. 2013;182:375-87. 